Laboratory Animals

47(3) 134-145

(C) The Author(s) 2013

Reprints and permissions:

sagepub.co.uk/

journalsPermissions.nav

DOI: 10.1177/0023677212473918

la.sagepub.com

(SAGE

\title{
A report of the Federation of European Laboratory Animal Science Associations Working Group
}

\author{
Dolores Bonaparte (Convenor) ${ }^{1,2}$, Paolo Cinelli ${ }^{3,4}$, \\ Eleni Douni ${ }^{5,6,7}$, Yann Hérault ${ }^{8,9,10}$, Alex Maas ${ }^{11,12}$, \\ Pirjo Pakarinen ${ }^{13,14}$, Matti Poutanen ${ }^{13,14}$, \\ Mirentxu Santos Lafuente ${ }^{15,16}$ and Ferdinando Scavizzi ${ }^{17,18}$
}

\begin{abstract}
The use of genetically-modified (GM) animals as research models continues to grow. The completion of the mouse genome sequence, together with the high-throughput international effort to introduce mutations across the mouse genome in the embryonic stem (ES) cells (www.knockoutmouse.org) facilitates an efficient way to obtain mutated mouse strains as research models. The increasing number of available mutated mouse strains and their combinations, together with the increasing complexity in the targeting approaches used, reinforces the need for guidelines that will provide information about the mouse strains and the robust and reliable methods used for their genotyping. This information, however, should be obtained with a method causing minimal discomfort to the experimental animals. We have, therefore, compiled the present document which summarizes the currently available methods for obtaining genotype information. It provides updated guidelines concerning animal identification, DNA sampling and genotyping, and the information to be kept and distributed for any mutated rodent strain.
\end{abstract}

\section{Keywords}

genotyping, husbandry, polymerase chain reaction, refinement, sampling

\footnotetext{
${ }^{1}$ SPCAL, Sociedade Portuguesa de Ciências em Animais de Laboratório

${ }^{2}$ Instituto de Medicina Molecular, Lisbon, Portugal

${ }^{3} \mathrm{SGV}$, Schweizerische Gesellschaft für Versuchstierkunde

${ }^{4}$ Institute of Laboratory Animal Science, University of Zürich, Zurich, Switzerland

${ }^{5}$ HSBLAS, Hellenic Society of Biomedical and Laboratory Animal Science

${ }^{6}$ Institute of Immunology, Biomedical Science Research Center 'Alexander Fleming', Athens, Greece

${ }^{7}$ Department of Agricultural Biotechnology, Agricultural University of Athens, Athens, Greece

${ }^{8}$ AFSTAL, Association Française des Sciences et Techniques de l'Animal de Laboratoire

${ }^{9}$ Institut Clinique de la Souris (ICS), INSERM U964, CNRS UMR7104, Université de Strasbourg, Illkirch, France

${ }^{10}$ Institut de Génétique de Biologie Moléculaire et Cellulaire (IGBMC), INSERM U964, CNRS UMR7104, Université de Strasbourg, Illkirch, France
}

\footnotetext{
${ }^{11}$ NVP, Nederlandse Vereniging voor Proefdierkunde

${ }^{12}$ Department of Cell Biology, Erasmus Medical Center, PO Box 2040, 3000 CA Rotterdam, The Netherlands

${ }^{13}$ ScandLAS, Scandinavian Society for Laboratory Animal Science

${ }^{14}$ Institute of Biomedicine and Turku Center for Disease Modeling, University of Turku, Turku, Finland

${ }^{15}$ SECAL, Sociedad Española para las Ciencias del Animal de Laboratorio

${ }^{16}$ Centro de Investigaciones Energéticas Medioambientales y Tecnológicas (CIEMAT), Madrid, Spain

${ }^{17}$ AISAL Associazione Italiana per le Scienze degli Animali da Laboratorio

${ }^{18}$ Consiglio Nazionale delle Ricerche, Istituto di Biologia Cellulare, European Mouse Mutant Archive, Monterotondo, Italy

\section{Corresponding author:}

D Bonaparte, Instituto de Medicina Molecular Animal Facility, Av. Prof. Egas Moniz, Edif. Egas Moniz, 1649-028 Lisboa, Portugal. Email: doloresbfafm.ul.pt
} 
As a starting point, we have decided to collect information on the genotyping practices applied in various European countries, in order to identify common problems and pitfalls faced and to better direct our recommendations. For this purpose, we have conducted a survey focused on genotyping-related procedures used. We have collected 158 responses from 25 countries (see Supplementary information http://lan.sagepub.com/ content/suppl/2013/05/28/0023677212473918.DC1/ LAN473918_Supplementaryd_data.pdf), which will be acknowledged in this report when appropriate. Only three replies were received with regard to rats, which seems to show that this species is still infrequently used for genetically-modified (GM) models. From the responses to this survey, we could conclude that the procedures are not harmonized among countries or even among different institutes within one country, and that the common practice is not always in accordance with the latest scientific findings.

\section{Collection of samples for genotyping}

Classical genotyping procedures for rodents rely on polymerase chain reaction (PCR) and Southern blot analyses of the DNA specimens obtained from tissue samples of individual animals. This approach is also supported by our survey, as shown in the Supplementary information (please see http://lan.sagepub.com/content/ suppl/2013/05/28/0023677212473918.DC1/LAN473918_ Supplementaryd_data.pdf).

Traditionally, the most widely used methods for obtaining the tissue material for genotyping are tail, ear, or toe biopsies. Because collecting such biopsies involves a given amount of discomfort for the animals, alternative methods for DNA collection have been proposed in order to reduce it. However, according to the survey of the present study (Table 1) these 'alternative' methods are not widely used, possibly due to problems related to accuracy, reproducibility, and practicability as identified in our survey and on comparative tests carried out by the members of this Working Group in their facilities.

Guidelines for the refinement and reduction of procedures involving GM rodents, also including recommendations for DNA sampling, have been published before by other Working Groups. ${ }^{1,2}$ One of the aims of the present task, thus, was to re-evaluate those guidelines in the light of new scientific evidence and to provide up-to-date information about the most adequate DNA sampling methods. These updated guidelines are general and will apply to the majority of strains. The recommended techniques should, however, be adjusted in case of specific phenotypic constraints that should render them impossible, inadequate, or more severe.

\section{Identifying the animals to genotype}

Animals have to be individually identified so that the determined genotypes can be assigned to the correct individuals. The FELASA Working Group on Rodent Identification has recently issued recommendations on this subject, which should be considered for detailed information. Coincidently, some reliable identification techniques provide a tissue sample as byproduct that can be used to extract DNA. Our primary recommendation is, therefore, that whenever there is the need to both identify an individual and collect a DNA sample for genotyping, a method should be chosen that meets both goals. This will avoid the use of two invasive techniques and will minimize handling, which is shown to be a major source of discomfort in this process. ${ }^{3,4}$ The survey performed by our group (see Table 1) showed that this rationale is not always followed by researchers and, thus, intensive education is needed in this regard.

\section{Collecting DNA samples for genotyping}

Overall, we recommend using the least invasive method that provides an adequate DNA sample in terms of quality and quantity to perform a robust genotyping procedure as described in the previous section. Whenever possible, this method should, at the same time, provide highly reliable identification.

Table 1. Methods used for sampling/genotyping and for identification both in newborns up to P17, and from P18 onwards, presented as percentage of survey responders.

\begin{tabular}{|c|c|c|c|c|}
\hline \multirow[b]{2}{*}{ Method } & \multicolumn{2}{|c|}{ Sampling/genotyping } & \multicolumn{2}{|c|}{ Identification } \\
\hline & P0-P17 & P18-adult & P0-P17 & P18-adult \\
\hline Tail biopsy & 42 & 79 & NA & NA \\
\hline Ear notch/punch & 5 & 43 & 8 & 64 \\
\hline Phalanx removal & 12 & 4 & 20 & 6 \\
\hline Blood & $<1$ & 13 & NA & NA \\
\hline Hair & 0 & $<1$ & NA & NA \\
\hline Faecal pellets & 0 & 0 & NA & NA \\
\hline Oral swab & 0 & 0 & NA & NA \\
\hline Ear tag & NA & NA & 4 & 33 \\
\hline $\begin{array}{c}\text { Tattoo/colour } \\
\text { marking }\end{array}$ & NA & NA & 15 & 6 \\
\hline Transponder & NA & NA & 0 & 6 \\
\hline $\begin{array}{l}\text { Isolation in a } \\
\text { single cage }\end{array}$ & NA & NA & 0 & $<1$ \\
\hline UV light & NA & NA & $<1$ & 0 \\
\hline No response & 43 & 6 & 53 & 8 \\
\hline
\end{tabular}

Total number of responders was 158. UV: ultraviolet, NA: not applicable. 
In addition to the invasiveness of the process in itself, the associated handling and restraining, which have been shown to be a major stress factor, should be considered. Moreover, species, strain, age of the animal and its phenotype must also be acknowledged. One should also make every effort to base judgement on scientific data, and interpret concepts such as invasiveness, pain and discomfort from the perspective of the animal, avoiding excessive anthropomorphization. ${ }^{5,6}$

Those sampling techniques that are also identification methods are thoroughly described in the Report of the Working Group on Rodent Identification. In these cases, our report will focus mainly on the aspects related to the collection of samples for genotyping.

Ear biopsy. Ear biopsy is a widely used method where a piece of tissue is removed from the mouse pinna. The method provides both a DNA sample and a means of identification, based on the location of the biopsy. The method seems not to induce major signs of discomfort ${ }^{3}$ and, because the ear is not very vascularized, bleeding is also minimal if present at all. Surprisingly, only $43 \%$ of the scientists in our survey reported using ear biopsy as a DNA sampling method on animals older than 17 days (Table 1). Moreover, 64\% used this method for identification purposes upon genotyping, indicating that around $20 \%$ of the scientists perform two invasive procedures where they could use just one.

There is a vast array of instruments that can be used for the procedure, ranging from small scissors to sophisticated ear punches with attached microtubes for easy tissue collection. The $2 \mathrm{~mm}$ version of the punchers is recommended for yielding enough DNA for PCR, while decreasing the risk of losing the identification mark by healing.

Before the ear is erect and when the pinna is still too small, this method is not possible or not accurate for identification purposes. Although varying with strain and individuals, the ear is usually sufficiently developed for this technique at around 14 days of age. After this threshold, ear biopsy should be the method of choice whenever there is a need for both permanent identification and collection of DNA for genotyping.

Tail biopsy. Tail biopsy consists of the amputation of a small portion of the distal tail. Despite being reliable and the most traditional tissue biopsy method for obtaining DNA for genotyping $(79 \%$ of the users in our survey use the method in mice over 17 days of age), this method does not provide any identification, thus requiring a concurrent procedure for this purpose. For this reason alone, it should be discouraged as first option when permanent identification is required unless an adequate DNA sample cannot be obtained by a more recommended technique.
If, however, this method is to be chosen, the following points should be considered:

1. Recent studies have shown that taking the biopsy on days 14 to 17 results in a less ossified sample which can be significantly shorter than what was recommended in the previous guidelines; $;, 8$

2. At this age, a $3 \mathrm{~mm}$ long biopsy will provide enough DNA for PCR. However, Southern blot analysis might require longer $(5 \mathrm{~mm})$ biopsies; $; 3,8,9$

3. Most mice react to tail snip and this reaction increases in intensity and number with age, probably due to vertebral maturation; ${ }^{7,10}$

4. Some guidelines ${ }^{2,8}$ and institutions recommend the use of analgesia or anaesthesia when a tail biopsy is taken at the age of 21 days or older. However, results of a recent study indicate that isoflurane anaesthesia did not reduce acute behavioural responses to tail biopsy in BALB/c and C57BL/6 mice. $^{10}$ Furthermore, it remains controversial whether local analgesia is effective and whether general analgesia or anaesthesia are advantageous in terms of global welfare as these procedures are not without adverse physiological impact. ${ }^{2,9,10}$

Because of the above, we recommend that tail biopsy should be used only when the techniques that account for both sampling and identification do not provide a tissue sample that is adequate for the genotyping protocol (e.g. Southern blot), or when the identification aspect of such techniques is redundant or not valid (e.g. the animal is already identified or requires a particular identification method other than ear marking, such as a transponder).

In cases where tail biopsy is used:

1. Mice should preferably be between 14 and 17 days old;

2. Sample should be no longer than $3 \mathrm{~mm}$, except when the genotyping technique is known to require a large amount of DNA;

3. A very sharp instrument should be used, with a clean, precise cutting gesture;

4. In case of bleeding, haemostasis should be enforced;

5. In older animals, the benefits of analgesia or anaesthesia should be evaluated.

Distal phalanx biopsy. Distal phalanx biopsy consists of the removal of the distal phalanx of a newborn animal, that is then used as a source of DNA. This method also provides a means of identification, and is described in detail in the literature ${ }^{11-13}$ and in the report of the FELASA Identification Working Group. 
A similar, non-refined method generally known as 'toe clipping' has been largely used in the past, regardless of the extent of the biopsy or the age of the animal. Owing to its mutilating aspect, that method was gradually set aside and is now discouraged or even prohibited in several European countries and institutions. In our survey, 17 of the 72 scientists reported Animal Ethics Committee restrictions regarding toe clipping at their sites of research, other than age-related.

Recent studies ${ }^{3,12-14}$ have shown that the refined distal phalanx biopsy of newborns, if properly performed, does not seem to affect mice more than tail or ear biopsy, neither in the short nor the long term. ${ }^{12,13}$ Conversely, tail biopsy has been shown to affect mice, at least in the short term. ${ }^{10,15}$

Although the technique is possible earlier, postnatal day (PND) 7 seems to be the preferred age for distal phalanx biopsy. At this age, toes are already well separated and easy to access, ${ }^{4,12,13}$ the ossification process is not yet completed, ${ }^{11}$ and the animals have reduced movements, which facilitates the accurate biopsy. There are national and institutional guidelines pointing out other ages as the limit, such as PND $12 .{ }^{16}$ However, we were unable to find scientific grounds for those.

With many studies requiring the genotyping of newborns and early genotyping becoming a growing trend, it is important to re-evaluate the sampling options available. At very early ages, ear sampling is not possible and tail biopsy requires concurrent marking techniques. In the light of current knowledge, distal phalanx biopsy (refined version) is, therefore, the method of choice to genotype newborns because it provides both identification and a source of good quality DNA on a sole intervention with minimum disturbance of the animals.

Accordingly, we recommend this method with the following safeguards:

1. At the time of the biopsy, the animals should be approximately seven days old;

2. Only the most distal phalanx of only one toe per paw should be removed;

3. No further biopsies should be performed.

We would like to take this opportunity to advocate early, pre-weaning genotyping. This approach has many advantages: the animals are easier to handle; some tissue samples yield more DNA as they are less ossified; and genotyping results are available before the weaning date, allowing for the better planning of experiments and management of the colonies. As an extra benefit, housing costs decrease as surplus animals can be managed before weaning time.

Blood. Blood can be used to extract DNA for genotyping, although it may present a few technical problems. ${ }^{17}$
It may also be a convenient method for genotype determination by using flow cytometry or microscopy-based techniques. ${ }^{18}$ In effect, $13 \%$ of the researchers who responded to our survey had used blood to determine the genotype (Table 1). However, blood sampling does not enable mouse identification. Hence, it can only be considered as a refinement in terms of sampling for genotyping in animals already identified, identified with non-invasive methods, or requiring an identification method that does not provide a DNA sample (e.g. transponders).

When this method is chosen, the amount of blood collected should respect the recommended volumes, and the collection technique should be the least invasive that is still appropriate for the required volume and genotyping method. ${ }^{19}$

Hair follicles. Hair follicles plucked from the animal can be used as a source of DNA for genotype identification. This technique would reduce the discomfort associated with the more invasive sampling techniques. $^{3,20}$ Unfortunately, its throughput is low and there is a high risk of cross-contamination between samples of different animals. ${ }^{3,20}$ Moreover, it does not provide a means of identifying the animals.

Owing to the above constraints, the technique is not appropriate for routine genotyping of large animal colonies. However, it can be considered for the following cases:

1. Low numbers of animals already identified, requiring an identification method that is not a sampling method (e.g. transponder) or that can be identified through a non-invasive method;

2. Older animals already identified, requiring an identification method that is not a sampling method (e.g. transponder) or that can be identified through a noninvasive method;

3. Re-sampling for confirmatory testing;

4. Colonies for which an invasive method can pose a threat (e.g. bleeding disorders).

Based on our survey, less than $1 \%$ of the scientists use hair follicle DNA for mouse identification (Table 1).

Colonic and rectal cells. Colonic and rectal cells, collected either by means of a rectal swab or scrape $e^{3,21}$ or from faecal pellets ${ }^{22-24}$ can be used as a DNA source for genotyping. While we would not think of rectal swabs or scrapes as non-invasive, the collection of faecal pellets could be a method to consider. Owing to possible DNA degradation, faecal pellets are to be obtained freshly, ${ }^{24}$ or at the latest within $24 \mathrm{~h}^{22}$ The faeces need to be individually collected and more than one faecal pellet per animal is normally required. 
For the above reasons, this method is not practical when sampling large numbers of animals. In addition, like hair plucking, the method does not provide a means for identification. Stool sampling may however be considered in the same situations described for hair sampling. None of those responding to our query reported using this method for genotyping.

Cells from the oral mucosa. To obtain oral cells for DNA sampling, the oral cavity may be scraped $^{25}$ or swabbed. ${ }^{26}$ Alternatively, the oral cavity can be flushed with a small amount of sterile water, although only a small amount of cells is retrieved by this technique from weanling mice. ${ }^{27}$ This method is widely used for human DNA collection, as it is virtually non-invasive in humans. However, there are reports of mice biting their tongues during the process, and the samples are often tinted by blood. ${ }^{3}$ Thus, the size and design of the collection tools should be carefully considered to keep the method non-invasive when applied in small rodents. Although this method could be considered useful for large colonies due to higher throughput and lower risk of contaminations when compared with the previous two methods, the expected low amount of DNA obtained is a limitation. Furthermore, the method also does not provide a means of identifying the animals. For this reason, we suggest that it should also be reserved for the situations listed for hair follicles and rectal cells.

None of the survey inquirers reported using this method.

\section{Summary of sampling recommendations}

In conclusion, when considering sampling for genotyping, we recommend the following:

- Work quietly and be gentle in order to minimize the stress associated with handling and restraint.
- Whenever possible, use a methodology that simultaneously identifies the animals and yields tissue for genotyping.

- Work carefully to avoid the need for repeating the procedures: identify the animals in a reliable way; collect the samples cautiously, and avoid crosscontamination.

- Whenever possible, opt for early genotyping.

- Collect the minimum amount of tissue that can still produce enough DNA for the selected genotyping method.

- Store and ship samples properly, to avoid DNA degradation. Consider extracting immediately and storing and/or shipping purified DNA instead of tissue.

Taking into consideration the age of the animals and the need to permanently identify them or not, Table 2 summarizes our recommended sampling methods for the most common situations. However, if the genotyping method requires a higher amount of DNA, or the recommended technique is inadequate due to physiological constraints of the model or scientific aspects of the project, another sampling method may have to be considered.

\section{Development of an optimized genotyping protocol}

An optimized genotyping protocol is essential to ensure fast and reliable mouse identification. PCR and Southern blot-based methods are currently by far the most commonly used methods. This was confirmed by our survey, with $92 \%$ of the respondents reporting the use of PCR followed by $10 \%$ using quantitative PCR, 9\% Southern blot/dot blot and 4\% single nucleotide polymorphism (SNP) detection as routine methods (see Supplementary information http://lan.sagepub.com/ content/suppl/2013/05/28/0023677212473918.DC1/ LAN473918_Supplementaryd_data.pdf).

Table 2. Recommended methods for the collection of samples for genotyping by polymerase chain reaction.

\begin{tabular}{llll}
\hline & $\begin{array}{l}\text { Sampling of animals } \\
\text { requiring permanent ID }\end{array}$ & $\begin{array}{l}\text { Batch sampling of animals not } \\
\text { needing permanent ID* }\end{array}$ & $\begin{array}{l}\text { Re-sampling, small numbers of } \\
\text { animals not needing permanent ID* }\end{array}$ \\
\hline $\begin{array}{l}\text { Day 5-Day } 7 \text { (Day } 7 \text { is preferred) } \\
\text { Day 8-Day } 13^{\dagger}\end{array}$ & $\begin{array}{l}\text { Distal phalanx biopsy } \\
\text { Distal phalanx biopsy }\end{array}$ & $\begin{array}{l}\text { Distal phalanx biopsy } \\
\text { Distal phalanx biopsy (best) } \\
\text { Tail biopsy ( } \leq 3 \mathrm{~mm} \text { ) }\end{array}$ & $\begin{array}{l}\text { Not applicable } \\
\text { Not applicable }\end{array}$ \\
Day 14-Day 17 & Ear biopsy & $\begin{array}{l}\text { Ear biopsy (best) } \\
\text { Tail biopsy ( } \leq 3 \mathrm{~mm} \text { ) }\end{array}$ & Hair/faeces/saliva Ear biopsy \\
After Day 17 & Ear biopsy & Ear biopsy & Hair/faeces/saliva Ear biopsy \\
\hline
\end{tabular}

*Also animals that, for experimental or institutional reasons, require an identification (ID) method that does not provide a sample (e.g. transponders).

$\dagger$ No information from the literature for this age interval - use good judgement.

FExcept for animals with previous ear marks that could be compromised by that ear biopsy. 
The advantages of the PCR-based methods are the speed and the low amount of DNA needed, which translate into a low amount of tissue required for sampling. Only 1-2 ng of DNA are needed for PCR, while Southern blot analysis requires microgram quantities of DNA. We provide below a few considerations for successful PCR and Southern-based genotyping of GM rodents.

\section{DNA purification}

Proper DNA purification is a critical step for setting up a PCR-based method for mouse identification, especially when a new genotyping protocol is established. After establishment of the method, the use of highly pure genomic DNA is not always necessary. This is also highlighted in our survey where only $18 \%$ of the respondents reported to the use of commercial kits for DNA purification as a routine (see Supplementary information http://lan.sagepub.com/content/suppl/2013/05/28/ 0023677212473918.DC1/LAN473918_Supplementaryd_ data.pdf).

Currently, there are several well-established methods available for extracting DNA from tissue biopsies. According to the experience of this Working Group and the results of our survey (see Supplementary information), the two most commonly used methods for tissue digestion are the use of proteinase $\mathrm{K}$ in the presence or absence of sodium dodecyl sulphate (SDS), and the use of alkaline lysis (HotSHOT method $^{28}$ ). In addition to these methods, there are several commercial DNA extraction kits available which tend to be easy to work with but are expensive to use. The survey confirmed that more than $75 \%$ of the respondents use proteinase $\mathrm{K}$ digestion for routine DNA isolation. We suggest three protocols which should deliver high-quality genomic DNA from tissue biopsies for PCR genotyping (see Supplementary information):

As crude tissue extract is typically used, DNA quality is not routinely analysed while genotyping GM mouse colonies. Supplementary Table S4 summarizes the most important DNA isolation protocols, the most common tissue sample and the respective procedure(s) for genotyping, i.e. PCR or Southern blot methods. Independent of the approach used for tissue collection, it is important to prevent contamination between the samples by means of proper working practices (using clean punching devices and forceps, avoiding contamination by hair, etc.) and to store samples properly to prevent DNA degradation. Where possible, it is also advisable to store part of the DNA to enable a repeat genotyping to be performed without the need for additional sampling. This is particularly important if the test is not optimized, or if problems are anticipated.

\section{Polymerase chain reaction}

This is a technique to amplify even a single copy of a particular DNA sequence, generating millions of copies. It is by far the most widely used method for genotyping routinely, a trend reflected in the results of the survey: $92 \%$ of the total number of responders reported using this method.

General considerations. For transgene detection, 150800 base pair long PCR products are typically used for efficient amplification. In multiplex PCR methods it is important to amplify fragments with similar lengths, with a preferred length difference of $50-100 \mathrm{bp}$, in order not to favour amplification of the shorter product. It is essential to set up a robust mutation-independent (control) PCR method for a single-copy gene expected to be present in any mouse strain. This is used as an internal control for successful DNA extraction, and to detect putative contaminants that interfere with DNA amplification. With the control genes, a product is added that should always be present, thus preventing false-negative results (Figure 1a and b). Examples of such genes and primer pairs which have been successfully used are provided in Table 3 .

For the genotyping of mutant mice produced with homologous recombination (such as knock-out and knock-in mice), typically three primers are used in PCR (see Figure 1c). In this case, the primers have to be designed so that the first two (forward and reverse) amplify a gene-specific region and the third primer (reverse) is specific for the selection cassette. In the normal situation, gene-specific primers are not able to amplify a product in the targeted locus (fragment is too long) and therefore amplify only the wild-type allele. Also, in this PCR strategy, products should have a difference in length of about $50-100 \mathrm{bp}$.

In the maintenance of large animal colonies, it is important that the PCR conditions are optimized in such a way that several genotyping PCRs can be performed simultaneously with the same programme. A detailed protocol for successful genotyping as well as a troubleshooting guide for PCR are provided as Supplementary document A (please see http://lan.sagepub. com/content/suppl/2013/05/28/0023677212473918.DC1/ LAN473918_Supplementaryd_data.pdf).

\section{Southern blot analysis}

Large amounts of DNA (7-15 $\mu \mathrm{g})$ of high purity, suitable for restriction enzyme cleavage, are needed for each digest. The resulting DNA fragments are separated by electrophoresis, transferred to membranes, and the transgenic product is visualized after hybridization with a specific labelled probe. The technique takes 


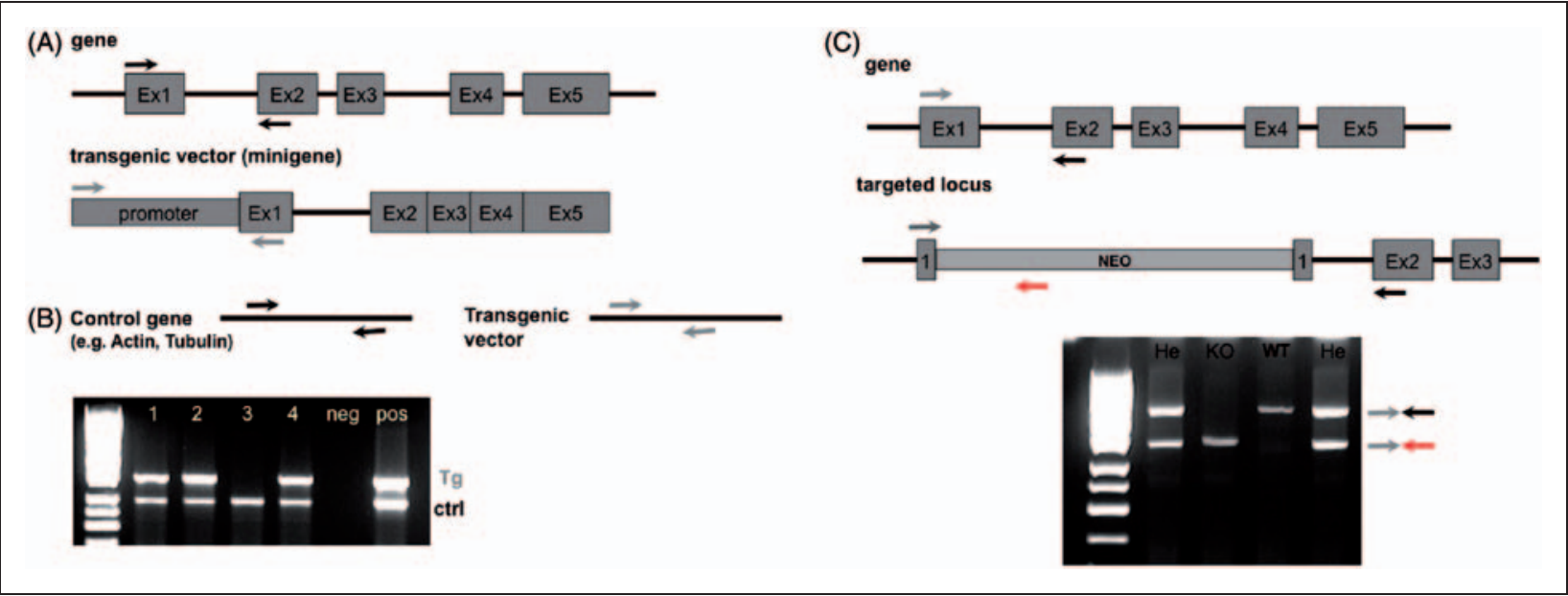

Figure 1. Use of control genes in polymerase chain reaction.

Table 3. Selection of genes and primers that can be used as internal controls.

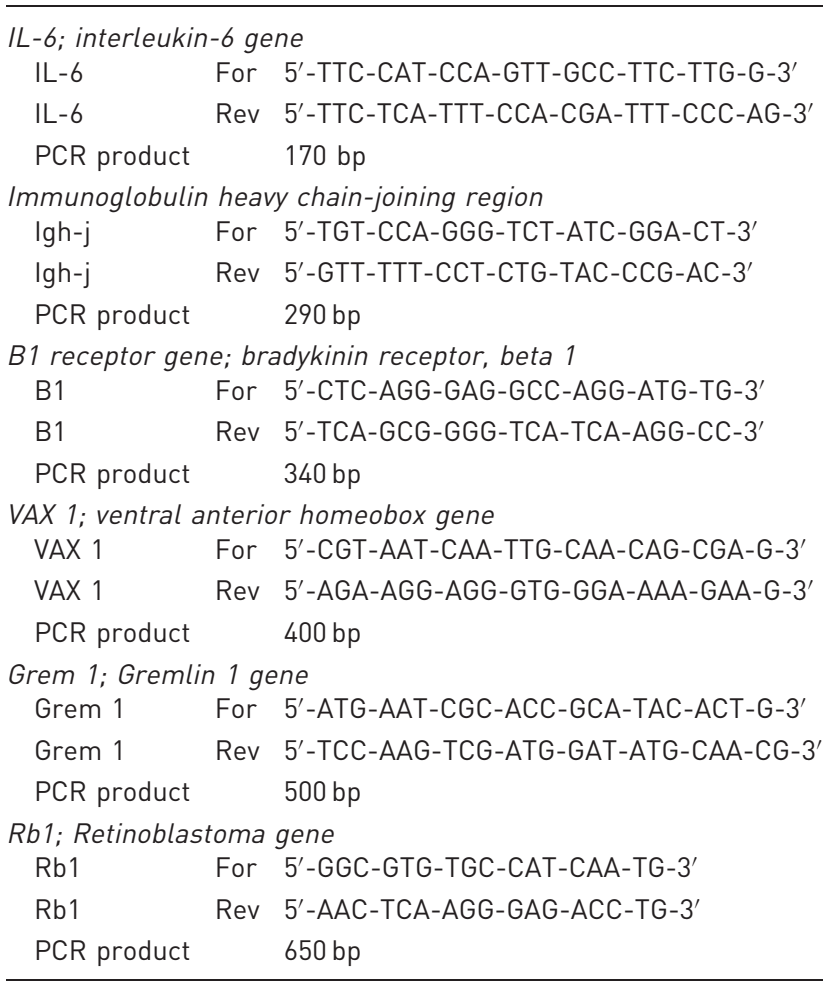

at least three days to complete and can be performed using non-radioactive labelling.

Southern blot requires more DNA and more tissue, but it is a required method in some instances. In the case that transgenics are generated by pronuclear injection of DNA, as it is the case for many lines carrying the Cre-expressing transgene, Southern blot is a powerful tool for determining the properties of transgene integration. It can be used: (1) to discriminate between lines when different integration sites occur in each line; (2) to determine the number of sites of integration; (3) to determine the transgenic copy number; (4) to verify the integrity of the transgene and the orientation of the tandem repeats; and (5) to detect homozygous individuals. Southern blot can also give information of the locus structure at the integration site and the overall integrity of the recombined transgene.

Thus, Southern blot is a useful technique to be used for the characterization of founders of a new transgenic line. When mutants are generated by gene targeting strategies, Southern blot is the gold standard for the identification of homologous recombination events in embryonic stem (ES) cells. In this case it is conceivable that, after obtaining germline competent chimeric animals upon injection of targeted ES cells into blastocysts, the mutations in the F1 generation are first tested by PCR, and upon expansion of the colony, only a representative number of animals is tested by Southern blotting, avoiding unnecessary collection of large tail biopsies.

\section{Other genotyping methods}

Quantitative realtime PCR. Realtime PCR allows the accumulation of the amplified product to be detected and measured as the reaction progresses. For the detection of PCR products, a fluorescent molecule is included in the reaction. This fluorescent molecule (DNA-binding dyes as SYBR Green or fluorescently labelled sequence-specific primers or probes as TaqMan) reports an increase in the amount of DNA with a proportional increase in the fluorescent signal, measuring the amount of amplified product in realtime.

In realtime PCR, the amount of amplification product is determined after each cycle, enabling a much more accurate quantification. It has been used for 
genotyping, ${ }^{29-31}$ for copy number detection of a transgene, ${ }^{32}$ and for determination of zygosity. ${ }^{33}$ However, for the latter purpose it should be used with caution as the method is not very reliable with high copy number of the transgene of interest. Realtime PCR also provides an accurate and sensitive method to determine the ultimate level of the Cre-specific gene disruption in conditional mutants with the Cre-loxP system. ${ }^{34}$

Genome-wide genotyping. Genome-wide genotyping enables scanning of the whole genome using polymorphic genetic markers for the identification of genetic differences between inbred or GM mice. Because genome-wide genotyping requires high-quality genomic DNA and adequate DNA quantity, genomic DNA is usually prepared from tail biopsies using a proteinase $\mathrm{K} / \mathrm{SDS}$ lysis buffer method followed by a phenolchloroform extraction and isopropanol precipitation. Subsequently, the genetic polymorphisms of test and control samples are amplified by PCR and ran on agarose gels or genetic analysers. The whole genome is scanned with high-density polymorphic markers, such as simple sequence length polymorphisms (SSLPs) and SNPs. We recommend using at least 2-3 genotyping markers, about $30 \mathrm{cM}$ in between each other, to assess the identity of the chromosomes.

SSLPs, also known as microsatellite markers, are serial di-, tri-, tetra- or penta-nucleotide repeats, where the number of repeat units varies among the different inbred strains. They are easily amplified by PCR using primers derived from unique sequences flanking the repeat units, and the length variance $(>4 \mathrm{bp})$ can be detected on $4 \%$ agarose gels. Usually the PCR product size of SSLP markers ranges from 80 to $250 \mathrm{bp}$. A dense genetic map of the mouse with numerous SSLPs that distinguish different inbred strains has been established ${ }^{35-37}$ which is freely accessible through various websites (Supplementary Table S5; please see http://lan. sagepub.com/content/suppl/2013/05/28/0023677212473 918.DC1/LAN473918_Supplementaryd_data.pdf) so that every molecular biology laboratory can easily establish its customized panel of SSLPs. Even though SSLP genotyping is easy and cost-effective, genome-wide genotyping has been further upgraded by the use of SNPs. ${ }^{38}$

SNPs are single base pair mutations that occur at a specific site in the DNA sequence and are the most common type of genetic variation among inbred strains. In addition, SNP is the genotyping method of choice for screening point mutations induced with the alkylating agent $N$-ethyl- $N$-nitrosourea (ENU). ${ }^{39,40} \mathrm{~A}$ plethora of SNP genotyping platforms is currently available. $^{41}$ High-throughput technologies genotype thousands of SNPs simultaneously, including Affymetrix GeneChip arrays, ${ }^{42}$ and Illumina BeadArray technology. ${ }^{43}$ Both platforms use the same basic principle of hybridization of genomic DNA fragments to a fixed probe. These microarray technologies require $250-750 \mathrm{ng}$ of genomic DNA per sample and only one sample is simultaneously tested for all markers per assay. Such methods offer a dense scan of the mouse genome with multiplex SNP genotyping and have mainly application in genetic mapping screens but are not considered cost-effective for genotyping a large panel of mice. Other customized methods for SNP genotyping, such as Pyrosequencing, ${ }^{44,45}$ Applied Biosystems TaqMan approach, ${ }^{46}$ and Sequenom MALDI-TOF mass $\operatorname{spec}^{47}$ are readily applied to a large number of samples, with relatively low running costs. Since SNP genotyping requires expensive equipments, reagents, experienced personnel, and significant analysis capabilities, it is usually offered as a service by institutional core facilities (Supplementary Table S5).

\section{Applications of the genome-wide genotyping methods}

Until recently, genome-wide genotyping was exclusively applied on genetic mapping for the identification of the causal mutation that was responsible for the observed phenotype. During the last decade, a forward genetics or phenotype-driven approach (phenotype to gene) was established in various research centres worldwide through genome-wide random ENU mutagenesis. This approach involves screening of thousands of mutagenized mice for mutant phenotypes and subsequent identification of the mutated gene. With the advances in genome sequencing and the discovery of thousands of polymorphisms in the mouse genome, the process of genetic mapping is now much more efficient and significantly less time-consuming than a decade ago.

Genome-wide genotyping has also important applications in the evaluation of the genetic purity and contamination of any mouse genetic background. ${ }^{38}$ Mutant lines maintained on an inbred background should be monitored regularly for evidence of genetic contamination due to accidental mismating. A genetic quality monitoring of breeders with approximately 50 genome-wide polymorphic markers offers detection of massive genetic contamination. However, subtle changes of the genetic background could be detected only by a dense scan of the genome with SNPs spreading all over the genome that are available from different providers (i.e. Affymetrix [www.affmetrix.com], Illumina [www.illumina.com]). Ultimately the next generation sequencing allows a complete overview of the mouse genome of interest and to compare with the original inbred genetic background (http://www.sanger. ac.uk/resources/mouse/genomes/) A successful genetic quality control monitoring system is essential for the maintenance of well-established strain characteristics 
and the reproducibility of experimental data between different groups over time, taking advantages of the whole genome genotyping techniques described previously.

Moreover, genome-wide genotyping accelerates dramatically the development of congenic strains which are mutant lines repeatedly backcrossed to an inbred strain. In this genome-based strategy, named speed congenics, offspring are genotyped with a genome-wide panel of genetic markers, and animals with optimal genotype (those that have retained the mutation and the largest proportion of recipient background) are selected for the next breeding step. The optimal breeding strategy appears to be a $10 \mathrm{cM}$ genome scan of $20-50$ males per backcross generation, which requires approximately 150 markers per mouse. The advantage of this approach is the fact that it minimizes the number of backcrosses required to establish a quality congenic mouse strain as early as with the fifth backcross generation $^{48,49}$ instead of the 10 generations that are needed if a traditional breeding scheme is followed. Speed congenics provides a fast, reliable and cost-effective way for backcrossing as it reduces by half the time that is required for the backcrossing of mutant lines, i.e. from 3 to 1.5 years and minimizes the animals that are needed for backcrossing.

\section{Relevant information for mutated rodent strains}

Most mutants have been generated on a hybrid genetic background, and it is well known how important the contribution of the genetic background is to the phenotype of a given mutation. Thus, it is essential to keep adequate records of detailed information on the type of mutation, methods for genotype identification, genetic background, etc. for all GM mouse strains generated, and to pass this information together with the mouse strain to any user and collaborator. An example of such a data sheet is included as a Supplementary document B (please see http://lan.sagepub.com/content/suppl/ 2013/05/28/0023677212473918.DC1/LAN473918_

Supplementaryd_data.pdf).

\section{Strain identification and type of mutation}

Every mutant strain must have a name providing precise information on the affected gene, the type of mutation, the parental inbred strain(s) and the code for the laboratory where this mutant originates from, which can be requested from the Institute for Laboratory Animal Research (ILAR) (Supplementary Table S5). Nomenclature rules are set by the International Committee on Standardized Genetic Nomenclature for Mice and the Rat Genome and Nomenclature
Committee. ${ }^{1}$ This nomenclature needs to be used in order to provide unquestionable information about the type of mutation introduced (Supplementary Table S5).

For any transgene, a schematic presentation describing in detail the transgene structure used is essential, including precise information about the gene regulatory region and the protein coding regions used. For transgenic mice, the insertion site is not typically analysed. However, if known, the flanking sequences of the integration site should be given.

In case of a gene trap, the genomic locus of the insertion should be provided together with the description of the targeted locus. This information is available from the ES cell providers, such as the International Gene Trap Consortium (Supplementary Table S5). If available, it is also essential to provide the information about the insertion site of the gene trap.

For a targeted insertion, a detailed description by one nucleotide resolution of the targeted locus, including positions of exons, introns, possible sites for loxPand Frt-sequences, probes and restriction enzyme sites used for confirming the targeted allele by Southern blotting, and PCR primers used for routine genotyping of the targeted locus should be provided, and preferably indicated also in a schematic drawing.

In general, it is preferable to attach the same information electronically in order to facilitate the analysis of the modified locus. If available, the original publication where the mouse model was described for the first time should also be included. Emphasis should also be given to report the site and type of a putative antibiotic selection gene and/or the reporter gene inserted into the locus. As an example, a schematic presentation of a hypothetical, multipurpose allele is presented in Figure 2.

\section{Identification of the genotype}

For efficient colony maintenance, and to promote scientific collaboration concerning the various mouse strains, protocols developed for the genotyping of rodents should be simple, easy and as robust as possible. Moreover, these demands should be met by a method causing minimal stress to the animals.

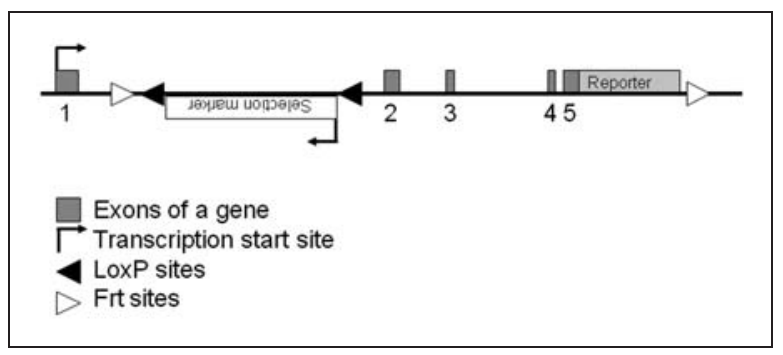

Figure 2. Example of a multipurpose gene knock-in. 
Once selected, the most appropriate genotyping protocol should be recorded along with the strain information, so that it could be reproduced without problems.

As mentioned previously, routine genotyping of mutated mouse strains are preferably performed by PCR. PCR would allow the distinction of wild-type, heterozygous and homozygous animals. However, in transgenic rodents this is not typically possible, due to the unknown integration site. In those cases, two independent primer pairs are designed to amplify a region with similar size: one specific for the transgene and another unrelated pair of primers are designed to demonstrate the proper amount and quality of the DNA in the specimen. This information should be adequately recorded and provided.

Equally important is the use of a reliable animal identification system, as no genotype information is valid without an unquestionable way to link it to an individual at any time and location within no matter how large animal facilities. It is, therefore, of the utmost importance that, not only are the mice well identified, but also the identification code and correspondent genotype are recorded and conveyed when transferring the animals to a different location.

Sometimes it is possible to identify the mutants by the phenotype. However, discriminating the mutant and wild-type littermates by the macroscopic appearance only is not always advisable. This is due to the fact that the phenotypic features might vary between the individuals, especially between generations and in the different genetic backgrounds.

\section{Genetic background}

The original genetic background (donor strain) onto which the mutation was originally introduced - e.g. embryo donors for the transgenic mouse production and the ES cell line used for homologous recombination or for the production of the gene trap clone should be described. Since phenotypic changes in the mutant rodent also depend on the genetic background, it is essential to provide a summary of the breeding history of the mouse model. International laboratory codes should be kept, as well as the terms Fxx, to indicate the number $(\mathrm{xx})$ of intercrosses, or Nyy to indicate the number (yy) of backcross generations from a donor to a new recipient background. ${ }^{1}$ As importantly, the gender of the carrier should be indicated as defined by the International Committee on Standardized Genetic Nomenclature.

Typically, backcrossing a mutation generated in a donor background for up to 10 generations into a new recipient background is considered necessary to produce a homogenous inbred mouse strain. However, it should be remembered that the genomic regions close to the genotyped mutation do not typically undergo homologous recombination; those regions are, thus, not backcrossed, containing alleles of the donor background for genes located at the vicinity of the mutation. If additional techniques are used, such as speed congenics, ${ }^{48}$ a description of the genetic markers used and located proximal to the mutation should be provided. Alternatively, performing a survey of the genetic background using high-density genetic markers such as SNP arrays developed by commercial companies would be recommended. ${ }^{42,43}$

The user needs to remember that some strains have particular characteristics (e.g. they are more prone to certain types of diseases). Thus, when using backcrossed mutants it is often needed to monitor unexpected influences of the genetic background. These possible changes should also be recorded along with the strain information.

\section{Husbandry characteristics}

To obtain and provide a short summary of the known phenotypic changes affecting the health and general fitness - and therefore, putatively required to apply for project approval by animal ethical committees and to adequately maintain the strains - is of the utmost relevance. Information on fertility of both genders, and any special considerations concerning the breeding strategies to be applied, should be monitored and conveyed. Similarly, information on the viability and fertility of both heterozygous and homozygous mutants should be recorded and provided for appropriate breeding strategy, housing, handling and maintenance of the mouse line.

Data on the history of the microbiological status of the rodent strain are of key importance for a smooth colony transfer between laboratories, and to recognize any health problems that might not be linked to the mutation itself. Indeed, even the bacterial gut flora could interfere with the mutation introduced (in particular for genes controlling metabolism or immunity) and impact the health or the response of the mutant.

\section{Concluding remarks}

The increasing number and complexity of GM rodent strains has determined a corresponding increase in the number and complexity of the procedures required for their successful generation, maintenance and use. It has also, fortuitously, resulted in increasing scientific studies aimed at refining these procedures in terms of effectiveness and of animal welfare. Unfortunately, many scientists are still making use of 'traditional' genotyping-associated procedures, even if these are not the 
most appropriate in terms of animal welfare or even of success.

With the present guidelines, we aim to further implement the concept of the $3 \mathrm{Rs}$ on genotyping-associated procedures, refining and harmonizing them in the light of the latest scientific findings, and according to the current scenario of animal research.

We believe that the proposed recommendations will lead to better animal welfare while improving scientific results and saving time and resources.

\section{Acknowledgements}

The Group would like to acknowledge Dr Pia Rantakari (University of Turku, Finland) and Professor F Claire Hankenson (University of Pennsylvania, USA) for their valuable and helpful collaboration in this project.

\section{References}

1. Rülicke T, Montagutelli X, Pintado B, Thon R and Hedrich HJ. FELASA guidelines for the production and nomenclature of transgenic rodents. Lab Anim 2007; 41: 301-311.

2. BVAAWF/FRAME/RSPCA/UFAW Joint Working Group. Refinement and reduction in production of genetically modified mice. Lab Anim 2003; 37(Suppl 1): S1-49.

3. Cinelli P, Rettich A, Seifert B, Bürki K and Arras M. Comparative analysis and physiological impact of different tissue biopsy methodologies used for the genotyping of laboratory mice. Lab Anim 2007; 41: 174-184.

4. Dahlborn K, Bugnon $\mathrm{P}$, Nevalainen $\mathrm{T}$, Raspa M, Verbost $\mathrm{P}$ and Spangeberg E. Report of the Federation of European Laboratory Animal Science Associations Working Group on animal identification. Lab Anim 2013; 47: 2-11.

5. Flecknell PA. Refinement of animal use - assessment and alleviation of pain and distress. Lab Anim 1994; 28 : 222-231.

6. Mayer $\mathbf{J}$. Use of behavior analysis to recognize pain in small mammals. Lab Anim (NY) 2007; 36: 43-48.

7. Hankenson FC, Garzel LM, Fischer DD, Nolan B and Hankenson KD. Evaluation of tail biopsy collection in laboratory mice (Mus musculus): vertebral ossification, DNA quantity, and acute behavioral responses. $J \mathrm{Am}$ Assoc Lab Anim Sci 2008; 47: 10-18.

8. NIH Guidelines for the genotyping of mice and rats [database on the Internet] 2002. See http://oacu.od.nih. gov/ARAC/documents/Rodent_Genotyping.pdf.

9. Arras M, Rettich A, Seifert B, Kasermann HP and Rülicke T. Should laboratory mice be anaesthetized for tail biopsy? Lab Anim 2007; 41: 30-45.

10. Hankenson FC, Braden-Weiss GC and Blendy JA. Behavioral and activity assessment of laboratory mice (Mus musculus) after tail biopsy under isoflurane anesthesia. J Am Assoc Lab Anim Sci 2011; 50: 686-694.

11. Han M, Yang X, Lee J, Allan CH and Muneoka K. Development and regeneration of the neonatal digit tip in mice. Dev Biol 2008; 315: 125-135.
12. Schaefer DC, Asner IN, Seifert B, Bürki K and Cinelli P. Analysis of physiological and behavioural parameters in mice after toe clipping as newborns. Lab Anim 2010; 44: 7-13.

13. Castelhano-Carlos MJ, Sousa N, Ohl F and Baumans V. Identification methods in newborn C57BL/6 mice: a developmental and behavioural evaluation. Lab Anim 2010; 44: 88-103.

14. Vachon P. Anatomical and histological observations of fore- and hind limb toes in adult mice after amputations performed at the age of two weeks. Can J Vet Res 1998; 62: 311-313.

15. Sorensen DB, Stub C, Jensen HE, et al. The impact of tail tip amputation and ink tattoo on C57BL/6JBomTac mice. Lab Anim 2007; 41: 19-29.

16. Tierversuchsverordnung - Verordnung des BVET über die Haltung von Versuchstieren und die Erzeugung gentechnisch veränderter Tiere sowie über die Verfahren bei Tierversuchen, 2010.

17. Winberg G. A rapid method for preparing DNA from blood, suited for PCR screening of transgenes in mice. PCR Methods Appl 1991; 1: 72-74.

18. Hellrung DJ, Rossi G and Link CJ. High-throughput fluorescent screening of transgenic animals: phenotyping and haplotyping. Cytometry A 2006; 69: 1092-1095.

19. Joint Working Group BFRU. Removal of blood from laboratory mammals and birds. First report of the BVA/FRAME/RSPCA/UFAW Joint Working Group on Refinement. Lab Anim 1993; 27: 1-22.

20. Schmitteckert EM, Prokop CM and Hedrich HJ. DNA detection in hair of transgenic mice - a simple technique minimizing the distress on the animals. Lab Anim 1999; 33: 385-389.

21. Lahm H, Hoeflich A, Rieger N, Wanke R and Wolf E. Identification of transgenic mice by direct PCR analysis of lysates of epithelial cells obtained from the inner surface of the rectum. Transgenic Res 1998; 7: 131-134.

22. Broome RL, Feng L, Zhou Q, et al. Non-invasive transgenic mouse genotyping using stool analysis. FEBS Lett 1999; 462: 159-160.

23. Kalippke K, Werwitzke S, von Hornung M, Mischke R, Ganser A and Tiede A. DNA analysis from stool samples: a fast and reliable method avoiding invasive sampling methods in mouse models of bleeding disorders. Lab Anim 2009; 43: 390-393.

24. Murgatroyd C, Bilko D and Spengler D. Isolation of high-quality DNA for genotyping from feces of rodents. Anal Biochem 2006; 348: 160-162.

25. Zhang YH, Huang BL, Eastman K, McCabe LL, MacLennan NK and McCabe ER. Mouth cell collection device for newborn mice. Mol Genet Metab 2006; 89: 164-167.

26. Meldgaard M, Bollen PJ and Finsen B. Non-invasive method for sampling and extraction of mouse DNA for PCR. Lab Anim 2004; 38: 413-417.

27. Irwin MH, Mofatt R.J and Pinkert CA. Identification of transgenic mice by PCR analysis of saliva. Nat Biotechnol 1996; 14: 1146-1148.

28. Truett GE, Heeger P, Mynatt RL, Truett AA, Walker JA and Warman ML. Preparation of PCR-quality mouse 
genomic DNA with hot sodium hydroxide and tris (HotSHOT). Biotechniques 2000; 29: 52, 54.

29. Hnatyszyn HJ, Podack ER, Young AK, Seivright R, Spruill G and Kraus G. The use of real-time PCR and fluorogenic probes for rapid and accurate genotyping of newborn mice. Mol Cell Probes 2001; 15: 169-175.

30. Wright KO, Murray DA, Crispe NI and Pierce RH. Quantitative PCR for detection of the OT-1 transgene. BMC Immunol 2005; 6: 20.

31. Terrell KA, Rasmussen TA, Trygg C, Bunnell BA and Buck WR. Molecular beacon genotyping for globoid cell leukodystrophy from hair roots in the twitcher mouse and rhesus macaque. J Neurosci Methods 2007; 163: 60-66.

32. Joshi M, Keith Pittman H, Haisch C and Verbanac K. Real-time PCR to determine transgene copy number and to quantitate the biolocalization of adoptively transferred cells from EGFP-transgenic mice. Biotechniques 2008; 45 : 247-258.

33. Sakurai T, Kamiyoshi A, Watanabe S, Sato $M$ and Shindo T. Rapid zygosity determination in mice by SYBR Green real-time genomic PCR of a crude DNA solution. Transgenic Res 2008; 17: 149-155.

34. Muller CC, Nourse JP, Nguyen TH and Crane DI. Quantitative genotyping of mouse brain-specific PEX13 gene disruption by real-time PCR. J Neurosci Methods 2009; 181: 73-81.

35. Detter JC, Nguyen QA and Kingsmore SF. Identification of novel simple sequence length polymorphisms (SSLPs) in mouse by interspersed repetitive element (IRE)-PCR. Nucleic Acids Res 1998; 26: 4091-4092.

36. Dietrich WF, Miller JC, Steen RG, et al. A genetic map of the mouse with 4,006 simple sequence length polymorphisms. Nat Genet 1994; 7(2 Spec No): 220-245.

37. Witmer PD, Doheny KF, Adams MK, et al. The development of a highly informative mouse simple sequence length polymorphism (SSLP) marker set and construction of a mouse family tree using parsimony analysis. Genome Res 2003; 13: 485-491.
38. Petkov PM, Cassell MA, Sargent EE, et al. Development of a SNP genotyping panel for genetic monitoring of the laboratory mouse. Genomics 2004; 83: 902-911.

39. Nolan PM, Peters J, Strivens M, et al. A systematic, genome-wide, phenotype-driven mutagenesis programme for gene function studies in the mouse. Nat Genet 2000; 25: $440-443$

40. Hrabe de Angelis MH, Flaswinkel H, Fuchs H, et al. Genome-wide, large-scale production of mutant mice by ENU mutagenesis. Nat Genet 2000; 25: 444-447.

41. Syvanen AC. Toward genome-wide SNP genotyping. Nat Genet 2005; 37(Suppl): S5-10.

42. Matsuzaki H, Dong S, Loi H, et al. Genotyping over 100,000 SNPs on a pair of oligonucleotide arrays. Nat Methods 2004; 1: 109-111.

43. Shen R, Fan JB, Campbell D, et al. High-throughput SNP genotyping on universal bead arrays. Mutat Res 2005; 573: 70-82.

44. Ronaghi M, Karamohamed S, Pettersson B, Uhlen M and Nyren P. Real-time DNA sequencing using detection of pyrophosphate release. Anal Biochem 1996; 242: 84-89.

45. Fakhrai-Rad H, Pourmand $\mathrm{N}$ and Ronaghi $\mathrm{M}$. Pyrosequencing: an accurate detection platform for single nucleotide polymorphisms. Hum Mutat 2002; 19: 479-485.

46. De la Vega FM, Lazaruk KD, Rhodes MD and Wenz MH. Assessment of two flexible and compatible SNP genotyping platforms: TaqMan SNP Genotyping Assays and the SNPlex Genotyping System. Mutat Res 2005; 573: 111-135.

47. Moran JL, Bolton AD, Tran PV, et al. Utilization of a whole genome SNP panel for efficient genetic mapping in the mouse. Genome Res 2006; 16: 436-440.

48. Markel P, Shu P, Ebeling C, et al. Theoretical and empirical issues for marker-assisted breeding of congenic mouse strains. Nat Genet 1997; 17: 280-284.

49. Armstrong NJ, Brodnicki TC and Speed TP. Mind the gap: analysis of marker-assisted breeding strategies for inbred mouse strains. Mamm Genome 2006; 17: 273-287. 